\title{
The size and symmetry of the lumbar multifidus muscles in Iranian industrial workers with and without chronic non-specific low back pain
}

\author{
Research Article
}

\section{Mohamad Ali Dayani ${ }^{1}$, Foruzan Ganji ${ }^{2}$, Hasan Rafiei ${ }^{3}$, Zahra Arshadi ${ }^{4}$, Masoud Lotfizadeh ${ }^{2}$, Mostafa Madmoli ${ }^{5}$, Ahmadreza Rafiei ${ }^{*}$}

1. MD, Assistant professor of Radiology, Faculty of Medicine,

Shahrekord University of Medical Sciences, Shahrekord, Iran

2. Social Determinants of Health Research Center, Shahrekord University of Medical Sciences, Shahrekord, Iran

3. MD, faculty of medicine, Shahrekord University of Medical Sciences, Shahrekord, Iran

4. MSc in Medical Biotechnology, Cellular and Molecular research centre, School of medical biotechnology,

Shahrekord University of Medical Sciences, Shahrekord, Iran

5. Emergency Medical Technician, Gerash University of Medical Sciences, Gerash, Iran

6. MD, Assistant professor of Neurosurgery, Faculty of Medicine,

Shahrekord University of Medical Sciences, Shahrekord, Iran

\begin{abstract}
Background: There is no specific cause of low back pain for eighty- five percent of cases and they were classified as non-specific low back pain. In this study, the size and symmetry of the lumbar multifidus muscles in industrial workers with non-specific chronic low back pain and healthy workers were investigated. Materials and Methods: In a cross-sectional comparative study, sixty industrial workers were divided into two groups of chronic low back pain and healthy, based on medical history and physical test. Roland-Morris Low Back Pain Questionnaire were filled by the group with chronic low back pain and dimensions of lumbar multifidus muscles in the L4-L5 level were measured by ultrasonography and the atrophy and asymmetry of two sides were examined. Results: Average size of right muscle in patients ranged between 2.5 and 4.3 CM? Inch? with average of $3.6 \pm 0.43$ and size of left muscle was in the range of 2.8 to 4.7 with the average of $3.9 \pm 0.46$. Size of right Muscle in healthy group was between 3.6 and 4.9 with the average of $4.3 \pm 0.4$ and left muscle size in healthy group was in the range of 3.9 to 5.09 and average of $4.4 \pm 0.42(\mathrm{p}<0.001)$. The proportion of symmetry, in the patient group was less $(0.9)$ compared to those with no symptoms $(0.95)(\mathrm{p}<0.001)$. Conclusion: This study showed that the size of the multifidus muscle in patients with chronic non-specific low back pain at L4-L5 level was smaller compared to without back pain subjects on the basis of the average size of right and left muscle. Besides, in the patient group,
\end{abstract}

Keywords: Ultrasound, multifidus muscle, non-specific chronic low back pain.

\section{Introduction}

Low Back pain is one of the most common musculoskeletal disorders in individuals under 65 years, so that it is the most common cause of activity limitation in people younger than 45 years (1 . Sixty to eighty percent of adults are suffering from this disorder during their lifetime $(2,3$ (. it has been suggested $90 \%$ of people experience back pain in their lifetime. (4)It is likely to state that low back pain is the most common human disease after common cold and it is the second most common cause of doctor visit (5 .(In people aged between 45 and 65 years, low back pain (LBP) is the third degree after heart disease and rheumatoid arthritis. With a high prevalence and its different causes, LAP is

*Corresponding Author:

\section{Ahmadreza Rafiei,}

Faculty of Medicine,

Shahrekord University of Medical sciences,

Shahrekord, Iran.

Email: rafieiahmad10@yahoo.com still one of the mysteries of medicine. It is likely to state that the cause of most of LAPs is not still determined (6, 7). About $60 \%$ of these patients affected again one year after the first occurrence of this pain $(7,8)$. The cost of treatment is very high in the case of chronic low back pain (9). Approximately one percent of the US population become disabled over time due to low back pain (10).

Ten to twenty percent? of patients with low back pain suffer from chronic pain syndrome (15). LAP is pain felt from groin (11). If LAP is accompanied by pain in the foot Radio cooler, it is called "Sciatalgia" or "Sciatica" (12). Patients with chronic low back pain are 73 to 77 percent of patients suffering from the waist disorders that in $85 \%$ of them, no specific cause have been found for low back pain $(13,14)$ and classified as nonspecific low back pain (15). This group dedicated eighty percent of the cost of back pain to themselves (16).

Beneck and his colleagues in 2005 compared the multifidus muscle in individuals with and without unilateral and bilateral chronic low back pain on 28 patients (14 patients, 14 healthy). The results of this 
study showed that despite the low level of disability, and level of similar activity in individuals in the control group, there was a significant bilateral localized atrophy multifidus (17).

Hides and his colleagues in 2008 in a study conducted on cricketers observed that cross-sectional area of multifidus muscle at L5 vertebra in 7 player having chronic back pain receiving strength training, had gone up compared with 14 healthy players (18).

Wall work and his colleagues in 2009 evaluated the effects of chronic low back pain in size and contraction of multifidus muscle on 34 patients with and without chronic low back pain by Ultrasound imaging. The results showed that the size and contraction of multifidus muscle in the L5 vertebra in patients with chronic low back pain was significantly smaller than the healthy group (19).

\section{Method}

It is a comparative cross-sectional study carried out in Fanavaran park of Shahrekord, Iran from October 2012 to March 2013. This comparative cross-sectional was carried out on 30 male workers with chronic nonspecific low back pain and 30 people with no symptoms employed workers in the industrial park of Fanavaran in Shahrekord. All subjects involved daily into industrial activity for more than 6 hours. Sampling method was simple Nonprobability convenient. All workers with low back pain participated in the study had a history of back pain for more than 3 months.

Patients' inclusion criteria include ages between 18 and 55, Employment for more than one year, Job satisfaction of workers, Non-specific low back pain for more than 3 months.

Inclusion criteria for healthy group includes ages between 18 and 50, Employment for more than a year, Employee satisfaction study, No back pain.

Exclusion criteria included history of the neurologic disease, neuromuscular, rheumatology, systemic diseases, history of hip or lumbar or thoracic fracture, lumbar spine surgery, cancer, spinal stenosis, diseases of internal organs, any known disorder of the spine such as scoliosis, spondylolisthesis or spondylolysis and addiction to drugs, mechanical back pain, Back pain caused by trauma, obvious neurological symptoms, history of severe trauma, history of neuromusculoskeletal and joint disorders, drugs affecting muscle size, any wounds or damaged skin around the ultrasound place, Mechanical Backaches, People with spinal deformity, History of abdomen or spine surgery, History of Neuromuscular and joint disorders.

At first, all volunteers participating in the study were informed of the objectives and empirical aspects of study and the consent form were signed by the participants after studying consent form.

Anthropometric characteristic including height and weight were measured and recorded by routine measurement tools. The BMI was calculated as weight divided by squared height. Information on the severity of back pain was registered in visual analog scale questionnaires (Visual Analogue Scale: VAS back pain) and disability resulting from LAP in daily activities recorded only by the patient group using a Roland-
Morris questionnaire (containing 24 questions and pointed from 0-24). In the present study, ultrasonography G5 model, Siemens brand Convex probe 3.5 and $5 \mathrm{MHz}$ frequency was used to assess the dimensions of the lumbar multifidus muscles. Patients lie down in prone position on the examination table so that their head located in the midline of the body and forehead on the inflatable Pillow.

Upper limbs placed in $90^{\circ}$ flexion and shoulders placed in relaxed state and a pillow was used to minimize the lumbar lordosis. To determine the vertebral level, L5 thorn-shaped appendages were detected by touching the base of the sacrum upward, other thorn-shaped appendages was found by the touching the L5 vertebra upwards. Then thornshaped appendages of L5 L4 was determined using a marker with lasting color before imaging. Then the volunteers were instructed to relax their paraspinal muscles, and then ultrasound gel was topically used and applicator was placed on the transverse thorn angles of the vertebrae. At this point, images registered where thorn-shaped appendages, the lamina and the outer edge of the multifidus muscle on both sides of the vertebral level was visible. To determine the edge of the deep multifidus muscles, echogenic of the vertebral lamina was used as an index. Bilateral Images was taken from multifidus muscle in the L4 and 15 levels.

Muscle dimensions include anteroposterior diameter (Anterior-Posterior Dimension: APD), lateral diameter (Lateral Dimension: LD), the size or the surface area (CSA) and the ratio of muscle shape (Shape ratio).

The maximum distance between the edge of the anterior and posterior muscle is called anterior-posterior diameter or thickness, and the maximum distance between the outer and inner edge of the muscle is named the muscle lateral diameter or width of muscle. These two diagonals are drawn perpendicular to each other. Muscle cross-sectional area of the environment or around of muscle used in this study to determine the size of the muscle is shown (Figure 1).

To determine the surface of the multifidus muscles, margin of muscle was followed by mouse embedded on the device and was drawn. For this purpose, the inner edge of the muscle was used as a fixed index. Thoracolumbar fascia was used to draw the posterior side and the echogenic vertebral lamina was used to draw anterior side.

To draw the inner edge, the fascia surrounding the multifidus muscle and fascia of separating it from the longissimus Erector spine muscles was used and to draw the inner edge, the shadow of sound waves reflecting from the tip and the outer edge of the thornshaped appendages were used. After drawing the edges of the multifidus muscle, software program of device was used to determine the cross-sectional area of muscle that can be seen in Figure 1.

To compare the symmetry of muscle size between the right and left sides between two groups, the ratio of smaller size to larger sizes can be used. In this formula, the more the symmetry of size between the two sides is greater, the ratio is closer to 1 . 
Sampling methods and sample size:

A total of 30 workers with chronic low back pain and 30 healthy workers between the ages of 18-55 was selected. Data was collected by low back pain RolandMorris questionnaire. The tool used to evaluate multifidus muscle was Siemens 3/5 convex ultrasound transducer probe for G5. Validity and reliability of this questionnaire was confirmed by Morteza'i in Iran and outside studies (20).

Data were analyzed with SPSS version 16, using mean and standard deviation and independent t-test. Difference between size mean between both the right and left and ratio of smaller to the larger size was calculated for both groups. $\mathrm{p}=0.05$ was statistically considered significant.

\section{Results}

In this study, 60 male workers employed in industrial centers were divided into two groups: workers with chronic non-specific low back pain $(n=30)$ aged between 22 and 52 years old and healthy workers (n $=30$ ) with age ranged between 23 to 41 years.

The average body mass index (BMI) of the patient group was $32.2 \pm 2.6$ and that of healthy group was $3.6 \pm 5.4$ as $(\mathrm{p}=0.68)$. Vas pain score in patients was between 1 and 10, with an average of $5.7 \pm 2.5$, respectively.

Besides, the amount of disability from back pain in patients based on Ronald-Morris questionnaires was between 2 and $15(62.5 \%-8.3)$, with an average of 8.6 \pm 4.4 .

Mean, standard deviation, minimum and maximum of data related to anthropometric characteristics of healthy and patient group, and vas pain score and disability among patients participating in the study are shown in Table 1-4. The size of lumbar multifidus muscle in both left and right sides were evaluated by ultrasonography in both groups. Size of Right muscle in patient group ranged between 2.5 and 4.3 with an average of $3.6 \pm 0.43$, and left muscle size ranged between 2.8 and 4.7 with an average of $3.9 \pm$ 0.46 .

Right Muscle size between in control group was between 3.6 and 4.9 with an average of $4.3 \pm 0.4$, and left muscle size in the same group ranged between 3.9 and 5.09 with an average of $4.4 \pm 0.42(p>0.001)$, the values of each are given in Table 2. The difference between the right and left muscle size in patient group was 0.3 and that of healthy group was 0.1 that based on the Pearson test, correlation coefficient ( $\mathrm{r}$ ) in patients 0.54 and the control group was 0.82 and estimated around $1.0(p<0.01)$. Overall, the average size of multifidus muscles in the patient group was significantly lower than that in healthy subjects $(p<0.001)$.

The proportion of size of smaller muscle to Larger or that of symmetry was less in healthy group compared to the patient group (0.9), so that the difference between the two groups was significant $(\mathrm{p}<0.001)$.

\section{Discussion}

Chronic low back pain is one of the most common musculoskeletal disorders in individuals under 65 years (1). For Eighty five percent of low back pain, no specific cause have been found and are classified as non-specific low back pain $(2,3)$. In this study, the size and symmetry of the lumbar multifidus muscles in industrial workers with chronic low back pain and healthy workers was discussed.

Size of Lumbar multifidus muscle on both the right and left sides were evaluated by ultrasonography in both groups. The difference between the right and left size in control and patient group was found. Overall, the average size of multifidus muscles in the patient group was significantly lower than that of the control group. The ratio of size of smaller muscle to Larger or that of symmetry was less in healthy group compared to the patient group (0.9), so that the difference between the two groups was significant.

Hides and colleagues (1994) also examined effect of unilateral acute low back pain on size of lumbar multifidus muscles in 26 patients and 51 healthy by ultrasonography and found that in the patient group, considerable asymmetry in multifidus muscle between the two sides of back was seen, In a way that muscle size in the painful area is smaller than other side and this reduction in the size is only related to one level of vertebra that at the same level of vertebra level in clinical examination, severe symptoms was tangible at that level ( 21$)$.

Atrophy of lumbar multifidus muscle is determined in the form of decrease in muscle size and change in the consistency of the muscle. This could be due to fibrotic changes, and infiltration of fat into muscle and tissue damage (22).

The results of this study revealed that similar studies conducted in this field. In a study by Beneck and colleagues, multifidus muscle size in subjects with and without unilateral and bilateral chronic low back was investigated. The results of this study show that despite the low level of disability, and similar level of activity in individuals in the control group, there were significant topical bilateral atrophy (20).

Hides and his colleagues in a study conducted on the cricketers, cross-sectional area of multifidus muscle in the L5 vertebra in 7 player with chronic back pain who received strength training, compared with 14 healthy players had gone up (22).

Wallwork and his colleagues, the effect of chronic low back pain in size and contraction of multifidus muscle using ultrasound imaging was assessed. The results showed that the size and contraction of multifidus muscle in the L5 vertebra in patients with chronic low back pain was significantly smaller than that of the healthy controls (23).

Arimi and his colleagues during a study was examined the symmetry of multifidus muscles in the neck in women with unilateral nonspecific chronic neck pain and healthy groups by ultrasonography. In the study, it was concluded that in the patient group, asymmetry of muscle size between the two sides and being smaller size of the painful muscle showed muscle atrophy in the involved side (24). 


\section{Conclusion}

This study showed that multifidus muscle size in the level L4-L5 lumbar in patients with chronic nonspecific low back pain was smaller than healthy group based on the average size of muscle on the right and left sides. Besides, in the patient group, muscle size was smaller than the other side, in a way that there was a clearly asymmetry between the two sides of the muscle in the spine. In addition, there was a difference between the two left and right back size in healthy subjects that can be attributed to their posture and daily habits. So asymmetry of muscle size between the two sides of the waist was naturally possible, but as it was observed in our study, in the presence of low back pain, asymmetry was added. The findings confirm results of other studies previously conducted on cervical and lumbar multifidus muscles by researchers using MRI and ultrasonography.

\section{Limitations}

1. Time consuming research (to find cases of patients who have inclusion criteria)

2. Difficulties in coordinating the transfer of workers to hospital

3. Cost of transferring of workers to hospital

\section{Acknowledgments}

The author of this article is grateful to all those who have collaborated with me in this article.

\section{Conflict of interest}

There are no conflicts of interest in this article.

\section{References}

1. Andersson GBJ. Epidemiological features of chronic low back pain. Lancet 1999; 56(7): $581 \mathrm{e} 585$.

2. Twomey LT, Taylor JR. Physical therapy of the low back. 3rd ed. Churchill Livingstone; 2002; 22(2): 190-198.

3. O'Sullivan P. Diagnosis and classification of chronic low back pain disorders: maladaptive movement and motor control impairments as underlying mechanism. Man Ther 2005; 10(4): 242-55.

4. Rucker KS, Cole AJ, Weinstein SM. Low back pain: a symptom-based approach to diagnosis and treatment. Boston: Butterworth-Heinemann; 2001; 62(2): 233-40.

5. Furlan AD, Imamura $M$, Dryden $T$, Irvin $E$. Massage for low back pain: An updated systematic review within the framework of the Cochrane Back Review Group. Spine (Phila Pa 1976). 2009; 34(16): 1669-84.

6. Frymoyer J. The Incidences and Costs of low Back Pain. J Orthopedic In Clinic. 1991; 22: 263-271.

7. Liebenson C. Rehabilitation of The Spin. J American of Orth Baltimore.1995: 22-3.

8. Andrea F, Marta I. Massage for Low Back Pain, An Updated Systematic Review Within the Framework of the Cochrane Back Review Group. Spine Journal. 2009; 34(16): 1669-1684.

9. Van Tulder MW, Koes BW, Bouter LM. A cost-ofillness study of back pain in The Netherlands. Pain 1995; 62(2): 233-40.

10. Courtney N, Towansend JR, Lawrence O, Spinal Injury in Neurosurgery. The Biological Basis of Modern Surgical Practice Philadelphia Saunders Company. 2001: 1518-1519.

11. Frymoyer JW. Back pain and sciatica. N Engl J Med 1988; 318(5): 291-300.

12. Carey TS, Garrett JM, Jackman AM. Beyond the good prognosis. Examination of an inception cohort of patients with chronic low back pain. Spine (Phila Pa 1976) 2000; 25(1): 115-20.

13. Indahl A, Velund L, Reikeraas O. Good prognosis for low back pain when left untampered. A randomized clinical trial Spine (Phila $\mathrm{Pa} 1976$ ) 1995; 20(4): 473-7.

14. Coste J, Delecoeuillerie G, Cohen de LA, Le Parc JM, Paolaggi JB. Clinical course and prognostic factors in acute low back pain: an inception cohort study in primary care practice. BMJ 1994; 308 (6928): 577-80

15. Main CJ, Watson PJ. Psychological aspects of pain. Man Ther 1999; 4(4): 203-15.

16. Waddell G. The back pain revolution. 2nd ed. Churchill Livingstone; 2004.; 20(6): 722-8.

17. Stude DE. Spinal Rehabilitation in: Orthop. Text Book Baltimore. Royal company. 1999: 5-43.

18. Hides j. Multifidus size and symmetry among chronic LBP and healthy asymptomatic subjects. Manual Therapy 13 (2008) 43-49.

19. Wallwork T. The effect of chronic low back pain on size and contraction of the lumbar multifidus muscle Man Ther. 2009 Oct;14(5):496-500. Epub 2008 Nov 21.

20. Mortezai SJ. The Oswestry disability index, the Roland and Morris disability questionnaire and the Quebec Back pain disability scale: translation and validation studies of the Iranian versions. Spine. 2006; 31: E454 - E459.

21. Hides JA, Stokes MJ, Saide M, Jull GA, Cooper $\mathrm{DH}$. Evidence of lumbar multifidus muscle wasting ipsilateral to symptoms in patients with acute/ subacute low back pain. Spine 1994; 19: 165-172.

22. Hides j. Multifidus size and symmetry among chronic LBP and healthy asymptomatic subjects. Manual Therapy 13 (2008) 43-49.

23. Lee H. Association between Cross-sectional Areas of Lumbar Muscles on Magnetic Resonance Imaging and Chronicity of Low Back Pain. Ann Rehabil Med 2011; 35: 852-859.

24. Arimi S, Soltani R. Evaluation of neck multifidus size and symmetry between people with chronic neck pain and healthy with ultrasonography. 2011;16(4):344-50. 


\section{Image 1: the cross-sectional of multifidus muscle}
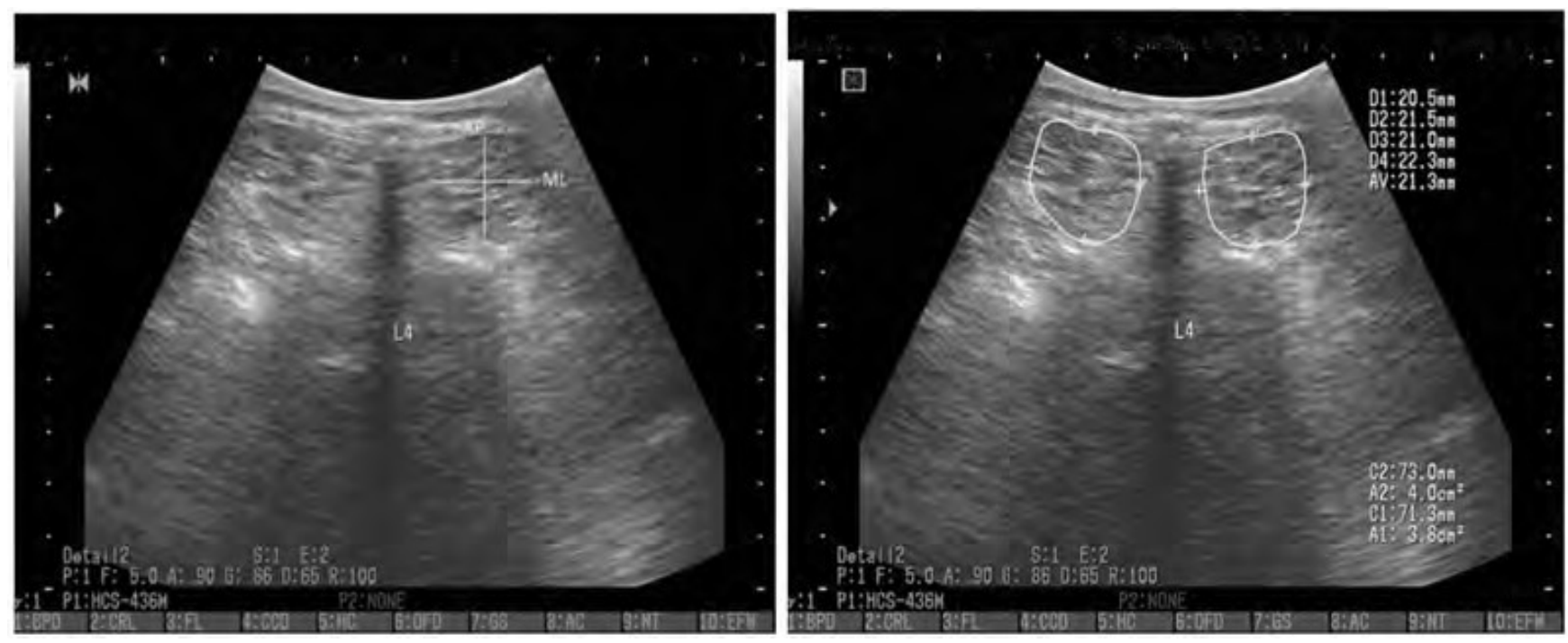

Figure 1: Mean, standard deviation, minimum and maximum and symmetry of multifidus muscle

Figure 2: presence or absence of atrophy in the right multifidus in healthy and patient groups
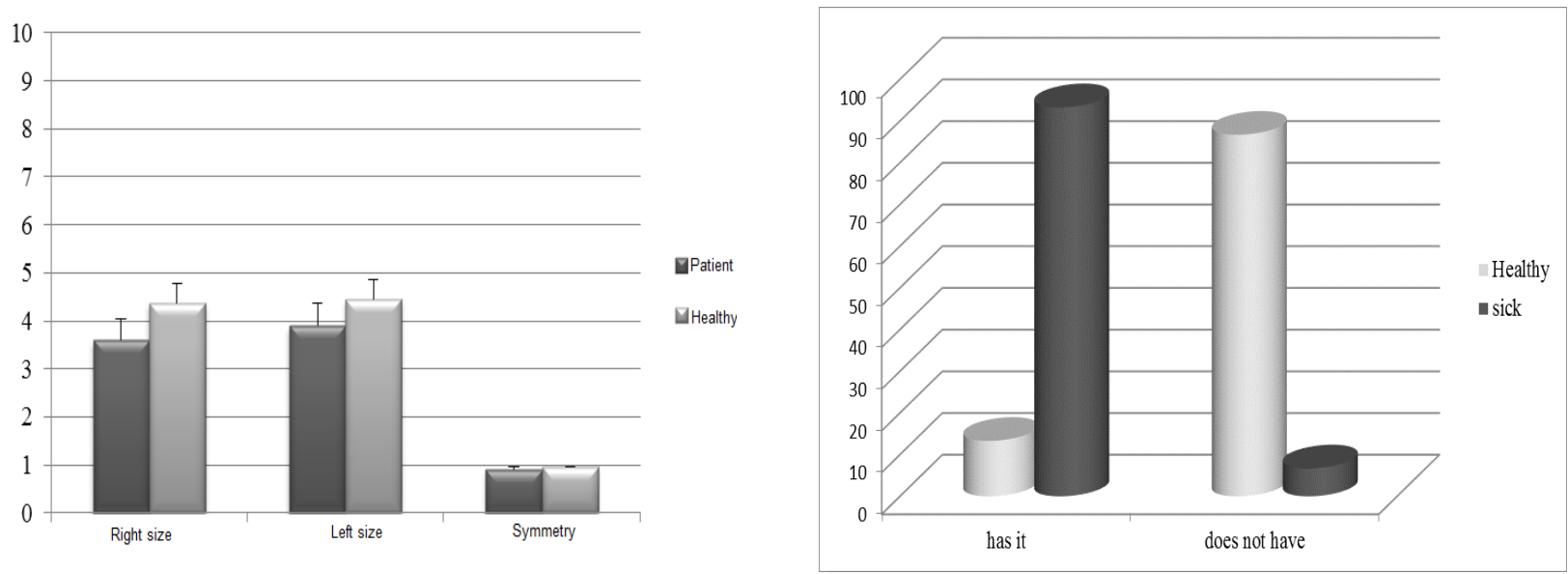

Figure 3: presence or absence of atrophy in the left multifidus in healthy and patient groups

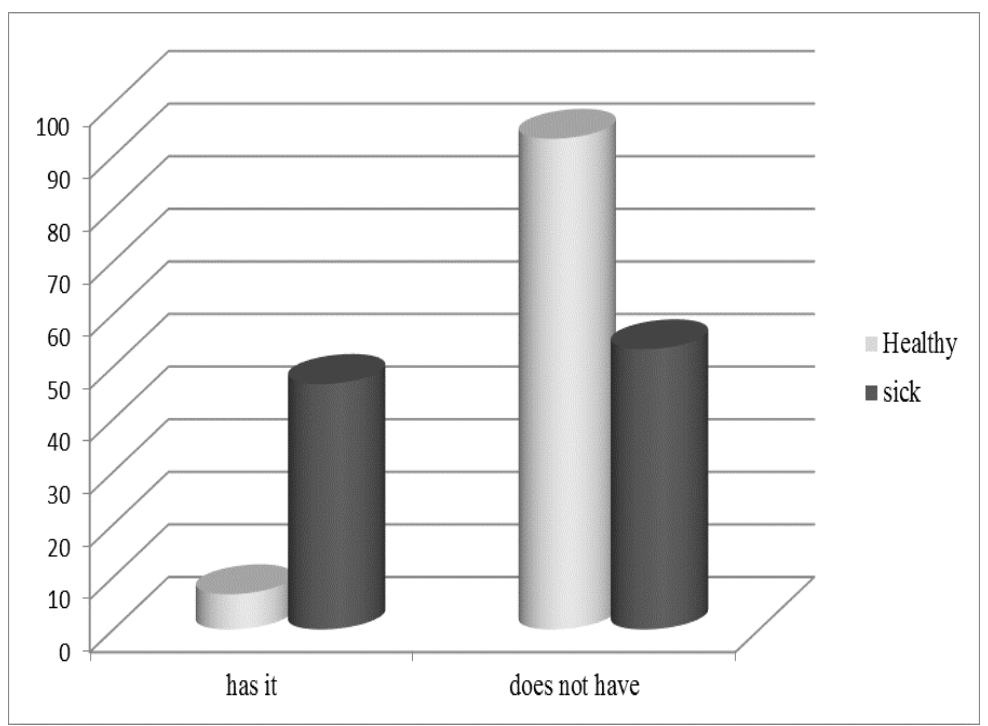




\section{International Journal of Ayurvedic Medicine, 2019, 10(2), 179-184}

Table 1: Mean, standard deviation, minimum and maximum of data related to anthropometric characteristics of healthy and patient group, and vas pain score and disability among patients

\begin{tabular}{|c|c|c|c|c|c|}
\hline Variables & Max & Min & Mean \pm SD & Groups & p value \\
\hline Age & 52 & 22 & $32.4 \pm 8.1$ & \multirow{6}{*}{ Patient } & \multirow{3}{*}{$\begin{array}{c}\mathrm{P}=0.30 \\
\text { Age }\end{array}$} \\
\hline Height & 185 & 170 & $178.1 \pm 5.2$ & & \\
\hline Weight & 84 & 57 & $73.7 \pm 7.5$ & & \\
\hline BMI & 27.4 & 0.917 & $23.2 \pm 2.6$ & & \multirow{3}{*}{$\begin{array}{c}\mathrm{p}=0.005 \\
\text { Height }\end{array}$} \\
\hline VAS & 10 & 1 & $5.7 \pm 2.5$ & & \\
\hline Disability \% & 0.562 & 0.38 & $8.06 \pm 4.4$ & & \\
\hline Age & 41 & 23 & $30.6 \pm 5.4$ & \multirow{4}{*}{ Healthy } & \multirow{2}{*}{$\begin{array}{l}\text { Weight } \\
\mathrm{p}=0.19\end{array}$} \\
\hline Height & 186 & 159 & $172.5 \pm 9.2$ & & \\
\hline Weight & 90 & 50 & $70.4 \pm 11.6$ & & \multirow{2}{*}{$\begin{array}{l}(\mathrm{BMI}) \\
\mathrm{p}=0.68\end{array}$} \\
\hline BMI & 30.30 & 20.55 & $26.4 \pm 1.97$ & & \\
\hline
\end{tabular}

Table 2: Mean, standard deviation, minimum and maximum and symmetry of multifidus muscle

\begin{tabular}{|c|c|c|c|c|c|c|c|c|}
\hline \multicolumn{2}{|c|}{} & \multicolumn{3}{|c|}{ Healthy group } & \multicolumn{3}{c|}{ Patient group } & $\begin{array}{c}\text { P-Value } \\
\text { (Independent t } \\
\text { test) }\end{array}$ \\
\cline { 2 - 9 } & Max & Min & Mean \pm SD & Max & Min & Mean \pm SD & $<0.001$ \\
\hline \multirow{2}{*}{$\begin{array}{c}\text { Muscle } \\
\text { size cm }\end{array}$} & Right & 4.9 & 3.6 & $4.3 \pm 0.4$ & 4.3 & 2.5 & $3.6 \pm 0.43$ & $<0.001$ \\
\cline { 2 - 9 } & left & 5.09 & 3.9 & $4.4 \pm 0.42$ & 4.7 & 2.8 & $3.9 \pm 0.46$ & $<0.001$ \\
\hline \multicolumn{2}{|c|}{ Symmetry } & 1 & 0.9 & $0.95 \pm 0.02$ & 0.99 & 0.78 & $0.9 \pm 0.07$ & $<$ \\
\hline
\end{tabular}

Table 3: presence or absence of atrophy in left and right multifidus muscle in healthy and patient groups

\begin{tabular}{|c|c|c|c|c|c|c|c|c|c|}
\hline \multirow{3}{*}{ Atrophy } & \multicolumn{4}{|c|}{ Healthy group } & \multicolumn{4}{|c|}{ patient group } & \multirow{3}{*}{$\begin{array}{c}\text { P-Value } \\
\text { Chi- } \\
\text { square } \\
\text { test }\end{array}$} \\
\hline & \multicolumn{2}{|c|}{ do not have } & \multicolumn{2}{|c|}{ have } & \multicolumn{2}{|c|}{ do not have } & \multicolumn{2}{|c|}{ have } & \\
\hline & $\%$ & Number & $\%$ & Number & $\%$ & Number & $\%$ & Number & \\
\hline right & 86.7 & 26 & 13.3 & 4 & 6.7 & 2 & 93.3 & 28 & $<0.001$ \\
\hline left & 93.3 & 28 & 6.7 & 2 & 53.3 & 16 & 46.7 & 14 & $<0.001$ \\
\hline
\end{tabular}

Table 4: Score, number and percentage of vas pain in the patient group

\begin{tabular}{|c|c|c|}
\hline Vas pain score & Number & Percent \\
\hline 1 & 2 & 3.3 \\
\hline 2 & 2 & 3.3 \\
\hline 3 & 2 & 3.3 \\
\hline 4 & 2 & 3.3 \\
\hline 5 & 6 & 10 \\
\hline 6 & 2 & 3.3 \\
\hline 7 & 10 & 16.7 \\
\hline 8 & 0 & 0 \\
\hline 9 & 0 & 0 \\
\hline 10 & 4 & 6.7 \\
\hline
\end{tabular}

